\title{
Distractor Generation for Chinese Fill-in-the-blank Items
}

\author{
Shu Jiang \\ Department of \\ Computer Science and Engineering \\ Shanghai Jiao Tong University \\ Shanghai, China \\ jshmjs45@gmail.com
}

\author{
John Lee \\ Department of \\ Linguistics and Translation \\ City University of Hong Kong \\ Hong Kong SAR, China \\ jsylee@cityu.edu.hk
}

\begin{abstract}
This paper reports the first study on automatic generation of distractors for fill-inthe-blank items for learning Chinese vocabulary. We investigate the quality of distractors generated by a number of criteria, including part-of-speech, difficulty level, spelling, word co-occurrence and semantic similarity. Evaluations show that a semantic similarity measure, based on the word2vec model, yields distractors that are significantly more plausible than those generated by baseline methods.
\end{abstract}

\section{Introduction}

The fill-in-the-blank item is a common form of exercise in computer-assisted language learning (CALL) systems. Also known as a cloze or gapfill item, a fill-in-the-blank item is constructed on the basis of a carrier sentence. One word in the sentence - called the target word, or key - is blanked out, and the learner attempts to fill it. The top of Table 1 shows an example carrier sentence whose target word is tiaojian 'condition'.

To enable automatic feedback, a fill-in-theblank item often specifies choices, including the target word itself and several distractors, as shown at the bottom of Table 1. Distractors need to be carefully chosen: they must be sufficiently plausible, but must not be acceptable answers. Literature in language pedagogy generally recommends the following criteria to authors of fill-in-the-blank items: a distractor should belong to the same word class and same difficult level, and have approximately the same length, as the target word (Heaton, 1989); it should collocate strongly with a word in the sentence (Hoshino, 2013); and it should be semantically related with the target word, ideally a

\footnotetext{
${ }^{1}$ This example is taken from (Liu, 2004).
}

\begin{tabular}{|c|c|}
\hline $\begin{array}{l}\text { 他因爲那裏的__ 不好, 戶戶 } \\
\text { 那裏上大學。 } \\
\text { He chose not to attend that u } \\
\text { because its__ are not good. }\end{array}$ & $\begin{array}{l}\text { 以不去 } \\
\text { versity }\end{array}$ \\
\hline $\begin{array}{l}\text { 1. 條件 tiaojian 'condition' } \\
\text { 2. 原因 yuanyin 'reason' } \\
\text { 3. 頻道 pindao 'channel' } \\
\text { 4. 條約 tiaoyue 'agreement' } \\
\text { 5. 函數 hanshu 'function' } \\
\text { 6. 因素 yinsu 'factor' }\end{array}$ & $\begin{array}{l}\leftarrow \text { Target word } \\
\downarrow \text { Distractors } \\
\quad \text { Human } \\
\quad \text { Baseline } \\
\quad+\text { Spell } \\
\quad+\text { Co-occur } \\
\quad+\text { Similar }\end{array}$ \\
\hline
\end{tabular}

Table 1: An example fill-in-the-blank item, with a carrier sentence with a blank (top); and six choices for the blank (bottom), including the target word (correct answer), and distractors generated by five different methods (see Section 4).

"false synonym" (Goodrich, 1977). An empirical study confirmed that distractors indeed tend to be syntactically and semantically homogenous (Pho et al., 2014).

To automate the time-consuming process of selecting distractors, there has been much interest in developing algorithms that, given a carrier sentence and a target word, can find appropriate distractors. To-date, most research effort on distractor generation for language learning has focused on English.

This paper presents the first attempt to automatically generate distractors in fill-in-the-blank items for learners of Chinese as a foreign language. In Section 2, we review related research areas. In Section 3, we present our datasets. In Section 4, we outline our criteria for distractor generation. In Section 5, we describe the evaluation procedure. In Section 6, we report evaluation results, show- 
ing that a semantic similarity measure based on the word2vec model yields distractors that are significantly more plausible than those generated by baseline methods.

\section{Previous work}

An algorithm for generating distractors must attempt a trade-off between two objectives. One objective is plausibility. Most approaches require the distractor and the target word to have the same part-of-speech (POS) and similar level of difficulty, often approximated by word frequency (Coniam, 1997; Shei, 2001; Brown et al., 2005). They must also be semantically close, which can be quantified with semantic distance in WordNet (Lin et al., 2007; Pino et al., 2008; Chen et al., 2015; Susanti et al., 2015), thesauri (Sumita et al., 2005; Smith et al., 2010), ontologies (Karamanis et al., 2006; Ding and Gu, 2010), or handcrafted rules (Chen et al., 2006). Another approach generates distractors that are semantically similar to the target word in some sense, but not in the particular sense in the carrier sentence (Zesch and Melamud, 2014). Others directly extract frequent mistakes in learner corpora to serve as distractors (Sakaguchi et al., 2013; Lee et al., 2016). Error-annotated Chinese learner corpora are still not large enough, however, to support broadcoverage distractor generation.

A second, often competing objective is to ensure that the distractor, however plausible, is not an acceptable answer. Most approaches require that the distractor never, or only rarely, collocate with other words in the carrier sentence. Some define collocation as $n$-grams in a context window centered on the distractor (Liu et al., 2005). Others also consider words elsewhere in the carrier sentence, for example those present in the Word Sketch of the distractor (Smith et al., 2010) or those that are grammatically related to the distractor in dependencies (Sakaguchi et al., 2013). Still others restrict potential distractors to antonyms of the target word, words with the same hypernym, and synonym of synonyms in WordNet (Knoop and Wilske, 2013).

To the best of our knowledge, there is not yet any reported attempt to generate distractors for learning Chinese vocabulary. The only previous work on Chinese distractor generation was designed for testing knowledge in the aviation domain, and leveraged a domain-specific ontology (Ding and
Gu, 2010).

\section{Data}

To facilitate our study, we compiled two datasets:

Textbook Corpus We collected 299 fill-in-theblank items, each with a target word and two to three distractors, from three Chinese textbooks (Liu, 2004, 2010; Wang, 2007). An analysis on this corpus confirms many of the criteria proposed in the literature: in $63 \%$ of the items, all distractors have the same POS as the target word; and in $45 \%$ of the items, at least one distractor shares a common character with the target word.

Wiki Corpus We extracted 14 million sentences from Chinese Wikipedia for calculating word frequency, similarity and co-occurrence statistics in the Candidate Generation step. We then performed word segmentation, POS tagging and dependency analysis on a subset of 5.5 million sentences with the Stanford Chinese parser (Levy and Manning, 2003) for use in the Candidate Filtering step.

\section{Approach}

We follow a two-step process where the first step, Candidate Generation, optimizes distractor plausibility; and the second step, Candidate Filtering, aims to filter out distractor candidates that are acceptable answers.

\subsection{Candidate Generation}

We implemented the following criteria for generating a ranked list of distractor candidates:

Baseline (Baseline) The baseline re-implements the criteria proposed by Coniam (1997): the distractor must have the same POS and the similar difficulty level as the target word. We extract all words in the Wiki corpus with the same POS, and then rank them by the proximity of their word frequency and that of the target word. In Table 1, for example, pindao 'channel' was chosen because, among all nouns, its word frequency is closest to that of the target word tiaojian.

Spelling similarity (+Spell) Many Chinese words contain multiple characters; two words that have one or more characters 
in common may be easily confusable for learners. This method requires the candidate to share at least one common character with the target word. In our running example in Table 1, tiaoyue 'agreement' was chosen because, among all words that contain the character tiao or jian (which combine to form the target word tiaojian), it has the most similar word frequency.

Word co-occurrence (+Co-occur) A distractor that often co-occurs with the target word may be easily confusable for learners. We ranked the candidate distractors according to their pointwise mutual information (PMI) score with the target word, as estimated on the Wiki corpus. In our running example in Table 1, hanshu 'function' was chosen because of its frequent co-occurrence with tiaojian 'condition'.

Word similarity (+Similar) Words that are semantically close to the target word tend to be plausible candidates. We ranked candidate distractors according to their similarity score with the target word. We obtained these scores by training a word2vec model (Mikolov et al., 2013) on the Wiki corpus. ${ }^{2}$ We opted for word2vec over thesauri or Chinese lexical databases such as HowNet because of its broader coverage. In the example in Table 1, the distractor yinsu 因素 'factor' was chosen because it has the highest similarity score with tiaojian in the word2vec model.

\subsection{Candidate Filtering}

A distractor is called "reliable" if it yields an incorrect sentence. This step aims to remove those candidates that are also acceptable answers, leaving only the reliable distractors. We do so by examining whether the distractor can collocate with words in the rest of the carrier sentence. The system examines the candidates in the ranked list produced by the Candidate Generation step (Section 4.1), and removes candidates that are rejected by both filters below:

Trigram The word trigram, formed by the distractor, the previous word and the following word in the carrier sentence, must not appear in the

\footnotetext{
${ }^{2}$ We trained a bag-of-words (CBOW) model of 400 dimensions and window size 5 with word2vec.
}

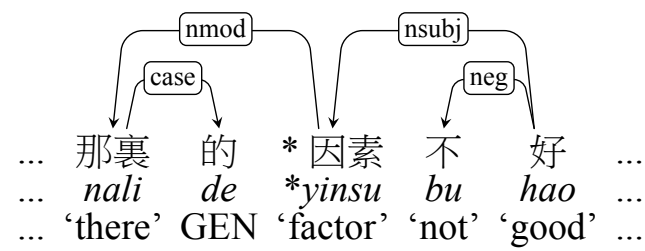

Figure 1: In the Candidate Filtering step (Section 4.2), candidate distractors whose dependency relations are attested in the corpus are rejected. To determine whether yinsu can serve as a distractor in the carrier sentence in Table 1, the system determines whether the dependency relations $\operatorname{nmod}(y i n s u$, nali) or nsubj(hao, yinsu) is attested in a large corpus of Chinese texts.

Wiki corpus. In the example in Figure 1, the trigram "de yinsu bu" must not be attested.

Dependency The Trigram filter alone might be too strict, since words that are grammatically related to the distractor may be further away. Among dependency relations in the parse tree of the carrier sentence, we extract all those with the distractor as head or child, and require that these relation must not be attested in the Wiki corpus. This filter is similar to the approach by Smith et al. (2010), but instead of the grammatical relations in Word Sketches, we consider all dependency relations. In our running example in Table 1 , the candidate 情况 qingkuang 'situation' was rejected because it is attested to serve as the subject of hao 'good'. The next distractor in the ranked list, yinsu 'factor', was chosen instead since it never served as the subject of hao 'good', and was never modified by the noun nali 'there'.

\section{Evaluation}

\subsection{Test data}

According to Da (2007), basic ability in Chinese news reading require a vocabulary of around 20,000 words. Among the target words in the Textbook Corpus, we selected 37 nouns and verbs such that they were roughly equally spaced among the 20,000 most frequent words in the Wiki Corpus.

For each of these 37 words, we generated distractors using each of the four criteria in Section 4 (Baseline, +Spell, +Co-occur, and +Similar). In addition, we randomly picked one 


\begin{tabular}{|l|c|}
\hline Method & Reliability \\
\hline Baseline & $100 \%$ \\
+Co-occur & $98.6 \%$ \\
+Spell & $93.2 \%$ \\
+Similar & $93.2 \%$ \\
\hline Human & $100 \%$ \\
\hline
\end{tabular}

Table 2: Reliability of the various distractor generation methods.

distractor from the corresponding fill-in-the-blank item in the Textbook corpus (Human). We thus have 37 items, each with six choices ${ }^{3}$ : one correct answer, and five distractors. Table 1 shows an example.

\subsection{Human annotation}

We asked two human judges, both native Chinese speakers, to annotate these choices, without revealing the target word. For each choice in the item, the judges decided whether it was correct or incorrect; they may identify zero, one or multiple correct answers. For an incorrect answer, they further assessed its plausibility as a distractor on a three-point scale: "Plausible" (3), "Somewhat plausible' (2)', or “Obviously wrong” (1).

The kappa for the human annotation is 0.529 , which is considered a "moderate" level of agreement (Landis and Koch, 1977). As a annotation quality check, we found that overall, in $6.8 \%$ of the times, a judge labels the target word as a distractor.

\section{Results}

\subsection{Reliability}

As shown in Table 2, the Baseline and + Co-occur methods performed best in terms of reliability: $100 \%$ and $98.6 \%$ of their respective distractors can be used. The +Spell and +Similar methods, at $93.2 \%$, were more prone to generating distractors that yield correct sentences. This is not unexpected since the +Similar method explicitly tries to find distractors that are semantically similar to the target word.

The reliability rate would have been lower if not for the Candidate Filtering step. The Trigram and Dependency filters rejected 16 of the 37 top candidates returned by the +Similar method. A post-

\footnotetext{
${ }^{3}$ Except that in 5 items, the + Co-occur and + Similar methods generated the same distractor; in another item, Baseline and +Co-occur generated the same distractor.
}

\begin{tabular}{|l|c|c|}
\hline Method & $\begin{array}{c}\text { Average } \\
\text { score }\end{array}$ & $\begin{array}{c}\text { Plausible or } \\
\text { somewhat plausible }\end{array}$ \\
\hline Baseline & 1.06 & $5.2 \%$ \\
+Co-occur & 1.27 & $8.6 \%$ \\
+Spell & 1.66 & $39.7 \%$ \\
+Similar & $\mathbf{1 . 7 6}$ & $\mathbf{4 6 . 6 \%}$ \\
\hline Human & $\mathbf{1 . 6 8}$ & $\mathbf{5 3 . 4 \%}$ \\
\hline
\end{tabular}

Table 3: Average scores, out of a 3-point scale (see Section 5.2), of distractors generated by the various methods in the human evaluation.

hoc analysis found that 11 of the 16 rejected candidates would indeed have been acceptable answers. The filters thus boosted the reliability rate by $30 \%$, at the cost of falsely rejecting 5 top-ranked candidates.

\subsection{Plausibility}

Table 3 shows the results on plausibility. Both the + Similar method ${ }^{4}$ and the + Spell method $^{5}$ outperformed the baseline, both in terms of the average score and the proportion of distractors considered at least somewhat plausible.

Distractors of the +Similar method have very competitive quality, scoring on average 1.76, slightly higher than the average score of the Human method (1.68). A qualitative review found that while the +Similar method can sometimes yield distractors that are even more plausible than those given by humans ${ }^{6}$, they are also more likely overall to be rated "Obviously Wrong", especially when the model fails to take into account word sense ambiguity: $53.4 \%$ of the Human distractors are rated Plausible or Somewhat Plausible, versus only $46.6 \%$ for the + Similar method.

\section{Conclusions}

We presented the first study on automatic generation of distractors for fill-in-the-blank items for learning Chinese. Evaluations showed that a semantic similarity measure, based on the word2vec model, offers a significant improvement over a baseline that considers only part-of-speech and word frequency, and achieves competitive plausibility in comparison to human-crafted items.

\footnotetext{
${ }^{4} p<0.001$, by McNemar's test.

${ }^{5} p<0.021$ by McNemar's test.

${ }^{6}$ Since we randomly selected one distractor out of three in the Textbook Corpus, the Human score reflects the average plausibility of the human-authored distractors, rather than the best one.
} 


\section{Acknowledgments}

This work is funded by the Language Fund under Research and Development Projects 2015-2016 of the Standing Committee on Language Education and Research (SCOLAR), Hong Kong SAR.

\section{References}

Jonathan C. Brown, Gwen A. Frishkoff, and Maxine Eskenazi. 2005. Automatic Question Generation for Vocabulary Assessment. In Proc. HLT-EMNLP.

Chia-Yin Chen, Hsien-Chin Liou, and Jason S. Chang. 2006. FAST: An Automatic Generation System for Grammar Tests. In Proc. COLING/ACL Interactive Presentation Sessions.

Tao Chen, Naijia Zheng, Yue Zhao, Muthu Kumar Chandrasekaran, and Min-Yen Kan. 2015. Interactive Second Language Learning from News Websites. In Proc. 2nd Workshop on Natural Language Processing Techniques for Educational Applications.

David Coniam. 1997. A Preliminary Inquiry into Using Corpus Word Frequency Data in the Automatic Generation of English Language Cloze Tests. CALICO Journal 14(2-4):15-33.

Jun Da. 2007. Reading News for Information: How Much Vocabulary a CFL Learner Should Know. In Andreas Guder, Jiang Xin, and Yexin Wan, editors, The Cognition, Learning and Teaching of Chinese Characters. Beijing Language and Culture University Press, pages 251-277.

Xiangmin Ding and Hongbin Gu. 2010. Automatic Generation Technology of Chinese Multiple-choice Items based on Ontology [in Chinese]. Computer Engineering and Design 31(6):1397-1400.

Hubbard C. Goodrich. 1977. Distractor Efficiency in Foreign Language Testing. TESOL Quarterly 11(1):69-78.

J. B. Heaton. 1989. Writing English Language Tests. Longman.

Yuko Hoshino. 2013. Relationship between Types of Distractor and Difficulty of Multiple-Choice Vocabulary Tests in Sentential Context. Language Testing in Asia 3(16).

Nikiforos Karamanis, Le An Ha, and Ruslan Mitkov. 2006. Generating Multiple-Choice Test Items from Medical Text: A Pilot Study. In Proc. 4th International Natural Language Generation Conference.

S. Knoop and S. Wilske. 2013. WordGap: Automatic Generation of Gap-Filling Vocabulary Exercises for Mobile Learning. In Proc. Second Workshop on NLP for Computer-assisted Language Learning, NODALIDA.
J. Richard Landis and Gary G. Koch. 1977. The Measurement of Observer Agreement for Categorical Data. Biometrics 33:159-174.

John Lee, Donald Sturgeon, and Mengqi Luo. 2016. A CALL System for Learning Preposition Usage. In Proc. ACL.

Roger Levy and Christopher D. Manning. 2003. Is it harder to parse Chinese, or the Chinese Treebank? In Proc. ACL.

Y.-C. Lin, L.-C. Sung, and M.-C. Chen. 2007. An Automatic Multiple-Choice Question Generation Scheme for English Adjective Understanding. In Proc. Workshop on Modeling, Management and Generation of Problems/Questions in eLearning, 15th International Conference on Computers in Education.

Chao-Lin Liu, Chun-Hung Wang, Zhao-Ming Gao, and Shang-Ming Huang. 2005. Applications of Lexical Information for Algorithmically Composing Multiple-Choice Cloze Items. In Proc. 2nd Workshop on Building Educational Applications Using $N L P$. pages $1-8$.

Jennifer Lichia Liu. 2004. Connections I: a Cognitive Approach to Intermediate Chinese. Indiana University Press, Bloomington, IN.

Jennifer Lichia Liu. 2010. Encounters I/II: a Cognitive Approach to Advanced Chinese. Indiana University Press, Bloomington, IN.

Tomas Mikolov, Kai Chen, Greg Corrado, and Jeffrey Dean. 2013. Efficient estimation of word representations in vector space. In Proc. ICLR.

Van-Minh Pho, Thibault André, Anne-Laure Ligozat, B. Grau, G. Illouz, and Thomas François. 2014. Multiple Choice Question Corpus Analysis for Distractor Characterization. In Proc. LREC.

Juan Pino, M. Heilman, and Maxine Eskenazi. 2008. A Selection Strategy to Improve Cloze Question Quality. In Proc. Workshop on Intelligent Tutoring Systems for Ill-Defined Domains., 9th International Conference on Intelligent Tutoring Systems.

Keisuke Sakaguchi, Yuki Arase, and Mamoru Komachi. 2013. Discriminative Approach to Fill-inthe-Blank Quiz Generation for Language Learners. In Proc. ACL.

Chi-Chiang Shei. 2001. FollowYou!: An Automatic Language Lesson Generation System. Computer Assisted Language Learning 14(2):129-144.

Simon Smith, P. V. S. Avinesh, and Adam Kilgarriff. 2010. Gap-fill Tests for Language Learners: Corpus-Driven Item Generation. In Proc. 8th International Conference on Natural Language Processing (ICON). 
Eiichiro Sumita, Fumiaki Sugaya, and Seiichi Yamamoto. 2005. Measuring Non-native Speakers' Proficiency of English by Using a Test with Automatically-Generated Fill-in-the-Blank Questions. In Proc. 2nd Workshop on Building Educational Applications using NLP.

Yuni Susanti, Ryu Iida, and Takenobu Tokunaga. 2015. Automatic Generation of English Vocabulary Tests. In Proc. 7th International Conference on Computer Supported Education (CSEDU).

Youmin Wang. 2007. 實用商務漢語課本（漢韓版） 準高級篇／高級篇. Commercial Press, Beijing.

Torsten Zesch and Oren Melamud. 2014. Automatic Generation of Challenging Distractors Using Context-Sensitive Inference Rules. In Proc. Workshop on Innovative Use of NLP for Building Educational Applications (BEA). 